\title{
Acero de alto silicio producido por inmersión en Al-Si y recocido de difusión $n^{(\cdot)}$
}

\author{
Tanya Ros-Yáñez*, Yvan Houbaert", Oldrich Schneeweiss" ${ }^{* *}$ Juan Asensio-Lozano*** \\ y Marta Prado-García y***
}

Resumen

Es difícil procesar aceros eléctricos de alto contenido en silicio $(>3,5 \% \mathrm{Si})$. Esto se debe principalmente, a problemas de fragilidad, aparición de grietas durante la laminación y oxidación. Sin embargo, existe un importante mercado para este tipo de acero en aplicaciones eléctricas debido a la favorable influencia que ejerce el $\mathrm{Si}$ sobre la magnetoestricción, las pérdidas eléctricas y la resistividad eléctrica. Como proceso alternativo, se sumergieron substratos de acero con $3 \%$ de silicio en una aleación hipereutéctica $\mathrm{Al}-25 \% \mathrm{Si}$, en un simulador de recubrimiento por inmersión en caliente. En los ensayos se utilizaron diferentes tiempos de precalentamiento y de inmersión. En la segunda fase de la investigación se llevaron a cabo recocidos de difusión en el mismo simulador para difundir una cantidad suficiente de $\mathrm{Si}$ en el acero. Las capas obtenidas y el substrato se caracterizaron mediante microscopía electrónica de barrido y análisis EDS observándose que:

- en todas las capas aparecen fases intermetálicas de acuerdo al diagrama ternario Fe-Si-Al.

- se forma la estructura ordenada $\mathrm{DO}_{3}\left(\mathrm{Fe}_{3} \mathrm{Si}\right)$, si el tiempo de inmersión y/o de difusión es suficiente, evidenciándose la difusión del silicio.

- por doble inmersión se obtienen grandes áreas de Si puro en la capa depositada.

- todavía no se lograron en los experimentos realizados concentraciones homogéneas de silicio en todo el espesor del substrato.

- cálculos teóricos demuestran que gradientes de Si en el acero también mejoran las propiedades magnéticas considerablemente.

Palabras clave Aceros eléctricos. Inmersión en caliente. Aceros de alto silicio.

\section{High silicon steel production through hot-dipping in Al-Si-alloy and diffusion annealing}

Abstract

It is difficult to process steel sheet with a high Si content $(>3.5 \% \mathrm{Si})$, mainly because of its brittleness and the appearance of cracks during rolling and oxidation. However, there is a market for this steel in electrical applications because of the favourable influence of $\mathrm{Si}$ on magnetostriction and electrical resistivity.

As an alternative process, $3 \% \mathrm{Si}$-steel substrates were coated with hypereutectic Al-Sialloys in a "hot-dip simulator" using different preheating and dipping times. The obtained layers were characterised by SEM and EDS analysis. Diffusion annealing experiments were performed to obtain sufficient amount of $\mathrm{Si}$ in the steel. It was observed that:

- intermetallic phases appear in the coating layers as according to the ternary $\mathrm{Fe}-\mathrm{Si}$ - $\mathrm{Al}$ diagram

- with a double dipping primary silicon crystals are formed in the surface layer

- the ordered $\mathrm{DO}_{3}$ structure is present if the dipping and/or diffusion time is long enough

- homogeneous silicon gradients in the whole substrate thickness have not yet been achieved

- theoretic calculations show that Si-gradient, also have beneficial effects on magnetic behaviour

Keywords Electrical-steels. Hot-dipping. High-silicon-steel

(•) Trabajo recibido el día 17 de julio de 2000 y aceptado en su forma final el día 20 de noviembre de 2000.

$\left(^{*}\right)$ Ghent University, Department of Metallurgy and Materials Science Technologiepark 9, B-9052 Zwijnaarde (Gent), Bélgica Tel. ++-32-9-2645762 - Fax.++-32-9-2645833 e-mail:Tanya.RosYanes@rug.ac.be,Yvan.Houbaert@rug.ac.be.

${ }^{* *}$ Institute of Physics of Materials, Academy of Sciences of Czech Republic Zizkovazz, CZ-616 63 Brno, Czech Republic e-mail:schneew@ipm.cz.

${ }^{(* *)}$ (3) Universidad de Oviedo. Escuela Técnica Superior de Ingenieros de Minas de Oviedo. C/ Independencia 13, Oviedo, España e-mail: jasensio@relay.etsimo.uniovi.es. 


\section{INTRODUCCIÓN}

Los aceros con alto porcentaje en silicio se usan en equipos para aplicaciones eléctricas, tales como motores, generadores, transformadores, etc. En los últimos años, los componentes eléctricos en estos equipos han comenzado a operar a frecuencias mayores de $400 \mathrm{~Hz}$ para lograr una mayor eficiencia y también la disminución en el tamaño de los mismos. Esto implica que el material seleccionado debe tener pocas pérdidas en el rango de altas frecuencias y una baja magnetoestricción.

Aceros al silicio con $3 \%$ Si se utilizan ampliamente en estos equipos, pero las óptimas condiciones, en términos de pérdidas, y la generación de ruido no han sido alcanzadas aún. Las propiedades magnéticas pueden ser mejoradas adicionando elementos de aleación como son silicio, en mayores proporciones, aluminio, manganeso y fósforo. Para aplicaciones eléctricas, las mejores propiedades se obtienen cuando se adiciona $6,5 \%$ de silicio en el acero. Con esta composición, la aleación alcanza una alta resistencia eléctrica y, en correspondencia, bajas pérdidas por Eddy-currents, mientras que la magnetoestricción se hace cero ${ }^{[1,4]}$. Sin embargo, el gran problema de altos porcentajes de silicio es la ductilidad extremadamente baja de la aleación resultante. Desde el punto de vista industrial es muy difícil laminar acero con $\mathrm{Si}>4 \%$, debido a la formación de una superestructura ordenada del tipo $\mathrm{DO}_{3}$ con composición $\mathrm{Fe}_{3} \mathrm{Si}$ (25\% atómico de silicio). En un proyecto Brite-Euram ${ }^{[5]}$ llevado a cabo en el laboratorio de Metalurgia de la Universidad de Gante en Bélgica, se logró laminar en caliente aceros con silicio hasta $6,5 \%$ obteniendo un espesor final de $1,0 \mathrm{~mm}^{[6]}$. Problemas de oxidación y de resistencia mecánica no permiten reducir más el espesor por laminación en caliente. La laminación en frío y en tibio se está estudiando en la fase actual de ese proyecto. Se han desarrollado muchos métodos adicionales para buscar una solución a este problema, como por ejemplo: técnicas de difusión del silicio utilizando pastas aplicadas en la superficie de la chapa ${ }^{[7,8]}$ y el método CVD (Chemical Vapour Deposition) desarrollado por la compañía japonesa $\mathrm{NKK}^{[9,10 \text { y } 11]}$.

La producción de acero de alto silicio por inmersión en un baño líquido de Al-Si hipereutéctico, seguido por un recocido de difusión es un método alternativo, desarrollado en nuestro laboratorio, para la obtención de esta clase de acero, evitando los problemas en la laminación derivados de la fragilidad del material ${ }^{[12,13,14}$ y ${ }^{15]}$. Simultá- neamente, la utilización de este método a escala industrial resultaría mas barato que el uso de métodos ya implantados ${ }^{[5,9,10}$ y 11$]$ como el CVD (Chemical Vapour Deposition).

En este estudio, una chapa de acero con $3 \%$ de silicio, todavía laminable hasta el espesor final deseado, se sumerge en un baño hipereutéctico de una aleación Al-Si. Después, se aplica un recocido de difusión para obtener una cantidad suficiente de aluminio y silicio en el acero, con el fin de aumentar la resistividad eléctrica y disminuir las pérdidas magnéticas.

\section{PARTE EXPERIMENTAL}

\subsection{Equipo utilizado}

Se trata de un simulador comercial para el estudio de procesos de recubrimiento en continuo por inmersión en caliente, de la marca RHESCA, que permite reproducir todas las etapas del tratamiento a partir de la chapa laminada en frío, tales como:

- simulación del proceso de recocido en continuo (tipo CAL ó CAPL) bajo atmósfera controlada

- recocido bajo atmósfera reductora para el control del estado de la superficie

- inmersión en baños fundidos de diferentes tipos y composiciones

- control del espesor del recubrimiento por toberas en forma de cuchilla (gas wiping system)

- enfriamiento controlado posterior a la inmersión

- tratamientos de recocido posterior (por ejemplo, del tipo "galvannealing")

El equipo consta de los siguientes elementos (Fig. 1):

- unidad de control del horno de fusión

- unidad de control del horno de infrarrojos para los recocidos

- controlador general de procesos por ordenador

- columna central consistente en:

- una cámara superior donde la muestra se introduce en el equipo y se puede realizar el enfriamiento rápido de la misma después de sumergirla. En la parte baja de esa cámara se encuentra el horno de infrarrojos donde se puede recocer la muestra antes de la inmersión y donde se puede realizar el recocido posterior de difusión.

- una cámara inferior, separada por una válvula de la cámara superior, en la que se encuentra el crisol con el metal fundido, 


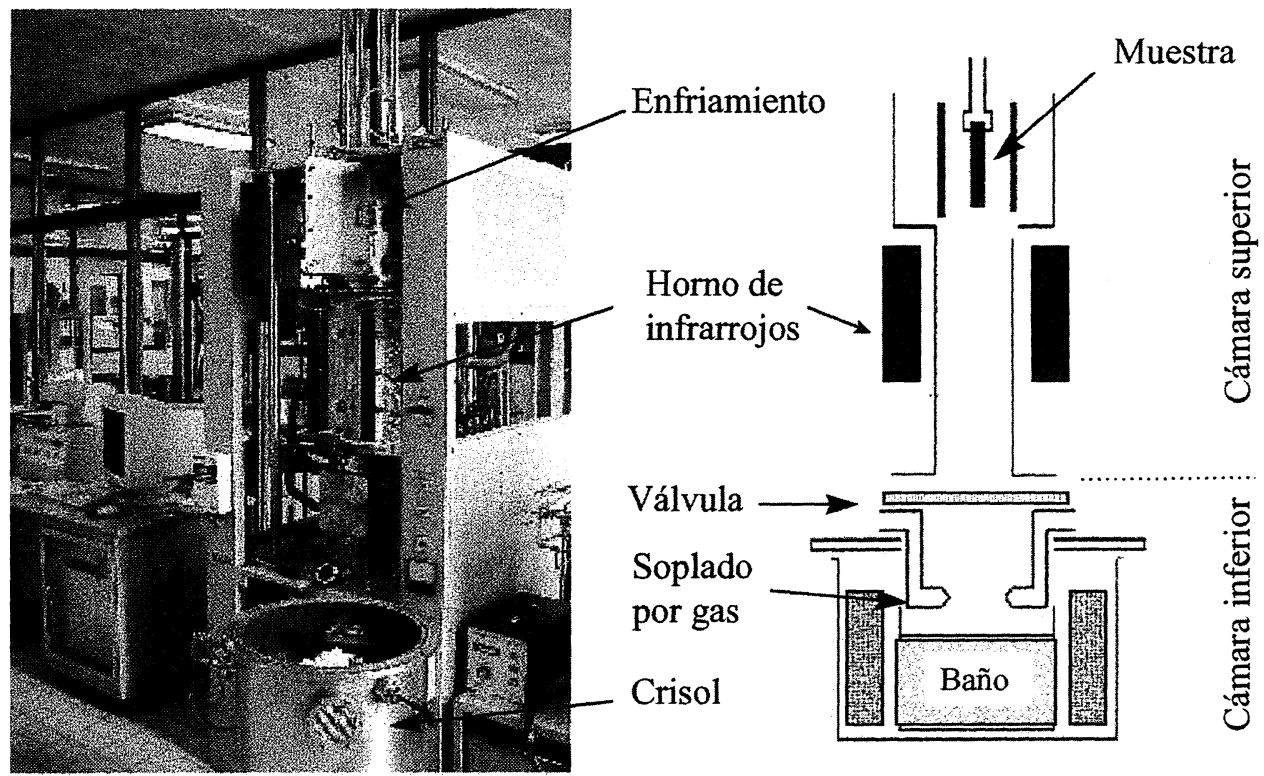

Figura 1. Simulador de recubrimiento por inmersión en caliente.

Figure 1. Hot dip simulator.

donde se sumerge la muestra. El control del espesor de la capa depositada se lleva a cabo a través del soplado de $\mathrm{N}_{2}$ y/o gas inerte por las toberas antes mencionadas.

\subsection{Metodología}

Las muestras, cuyas dimensiones son, generalmente de $200 \times 110 \mathrm{~mm}$, se introducen en la cámara alta del equipo, en una mordaza que va desplazándose por las diferentes secciones del simulador bajo atmósfera controlada. La temperatura de la muestra se monitoriza por medio de un termopar soldado a ella.

La muestra, limpiada previamente, se precalienta en el horno de infrarrojos hasta la temperatura deseada y bajo atmósfera reductora de $\mathrm{N}_{2}+5 \% \mathrm{H}_{2}$ en la cámara inferior del equipo. Se sumerge en la cámara inferior del equipo, durante un tiempo predefinido, en el baño líquido, sin soplar gas al salir del mismo. Después de la inmersión, la muestra se desplaza nuevamente hacia el horno de infrarrojos donde puede hacerse el recocido de difusión y, finalmente, se enfría en la cámara superior y se retira del equipo.

En la figura 2 se observa el esquema de la inmersión de la muestra en el baño líquido del simulador. El eje de las abcisas representa el tiempo $(t)$ y el de las ordenadas la profundidad $(x)$ a la que se encuentra la muestra en el baño. La línea continua representa el recorrido de la misma, indicando que la muestra se introduce con una cierta velocidad

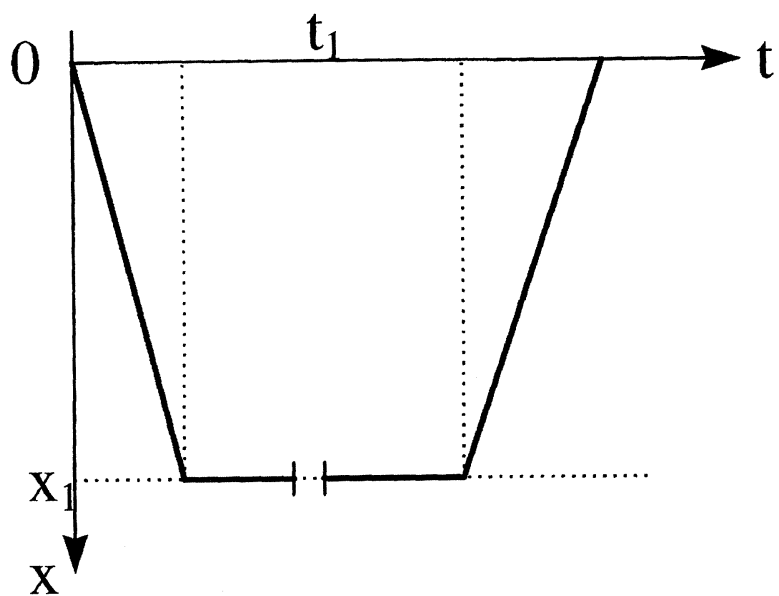

Figura 2. Esquema de inmersión de la muestra en el baño líquido del simulador de recubrimiento en caliente.

Figure 2. Schematic representation of sample dipping.

en el baño y una vez que llega al fondo, situado a una distancia $\left(x_{1}\right)$ de la superficie, permanece allí un determinado tiempo $\left(t_{1}\right)$ el cual se denomina tiempo de inmersión.

Un ordenador establece las velocidades de transferencia entre cada una de las etapas, la velocidad de calentamiento y enfriamiento, el tipo de tratamiento y la composición y tipo de atmósfera durante el proceso. A su vez, se registran los parámetros de operación del conjunto y el perfil térmico real.

La idea inicial del trabajo fue usar el simulador de inmersión en caliente para depositar una capa de silicio puro en un substrato de acero al silicio, con $3 \% \mathrm{Si}$, aproximadamente, para difundir luego 
el silicio en el interior de la chapa durante el recocido. Debido a que el silicio puro tiene un alto punto de fusión $\left(1.414{ }^{\circ} \mathrm{C}\right)$, se seleccionó una aleación hipereutéctica de aluminio-silicio para reducir la temperatura del baño líquido tanto en el simulador como en el posible proceso industrial.

Estudios previos realizados en el laboratorio con aleaciones hipereutécticas Al-Si mostraron que el mejor baño correspondía a una aleación de 23 a $25 \%$ de Si, considerando las características térmicas del equipo y la calidad de las capas depositadas. En dichos experimentos la temperatura óptima del baño se determinó variando la temperatura del mismo en pasos de $25^{\circ} \mathrm{C}$, desde $650^{\circ} \mathrm{C}$ hasta $825^{\circ} \mathrm{C}$. La mejor temperatura de acuerdo a los resultados obtenidos fue encontrada a 800 ${ }^{\circ} \mathrm{C}^{[12]}$.

\subsection{Materiales y parámetros utilizados}

Para el presente estudio se seleccionó como substrato un acero eléctrico industrial de grano orientado con un espesor de $0.35 \mathrm{~mm}$, cuya composición química se indica en la tabla I. Este acero se elaboró según la ruta convencional de fabricación ${ }^{[1,2]}$, incluyendo la descarburación después del segundo ciclo de laminado en frío, pero sin llevar a cabo el recubrimiento de la chapa con película de $\mathrm{MgO}$ ni el recocido de recristalización secundaria con la formación de la capa vidriosa de forsterita (silicato de magnesio) ${ }^{[2]}$. De este modo, fue posible conservar una superficie limpia y capaz de aceptar el depósito de silicio desde la fase líquida en la inmersión ${ }^{[13,14,15]}$.

Se realizaron cuatro series de experimentos donde se modificaron los siguientes parámetros tecnológicos: la temperatura de precalentamiento de la muestra, el tiempo de inmersión, la temperatura y los tiempos de recocido de difusión. También, en algunas muestras, se realizó una segunda inmersión después de un recocido intermedio. La temperatura del baño líquido se mantuvo siempre constante a $800^{\circ} \mathrm{C}$.

La temperatura de las muestras se varió entre 650 y $850^{\circ} \mathrm{C}$, y el tiempo de inmersión entre 0,1 y

Tabla I. Composición química del substrato antes de sumergir (\% en masa)

Table I. Chemical composition of the substrate before dipping (in wt \%)

\begin{tabular}{ccccccccc}
\hline $\mathrm{C}$ & $\mathrm{Si}$ & $\mathrm{Mn}$ & $\mathrm{P}$ & $\mathrm{S}$ & $\mathrm{Al}$ & $\mathrm{Nb}$ & $\mathrm{Ti}$ & $\mathrm{N}$ \\
0.003 & 3.20 & 0.14 & 0.016 & 0.018 & 0.016 & 0.005 & 0.003 & 0.0008 \\
\hline
\end{tabular}

600 s. El tratamiento térmico posterior de algunas de las muestras se limitó a un enfriamiento rápido por soplado de nitrógeno. En cambio, a otras se les aplicó un recocido con temperaturas entre 700 y $800^{\circ} \mathrm{C}$, durante $60 \mathrm{~s}$, seguido de un enfriamiento controlado a $-20^{\circ} \mathrm{C} / \mathrm{min}$ hasta $300^{\circ} \mathrm{C}$ en principio lento y, posteriormente, soplando nitrógeno.

Las capas obtenidas y el substrato se caracterizaron mediante microscopía electrónica de barrido y análisis EDS (Energy Dispersive System). Las muestras se prepararon usando pulido mecánico con pasta de diamante de 0,25 $\mu \mathrm{m}$ y sin ataque químico.

\section{RESULTADOS EXPERIMENTALES}

Durante la inmersión del substrato en el baño líquido se forman diferentes capas de compuestos $\mathrm{Fe}$-Si-Al. Con el fin de predecir qué fases pueden formarse sobre el substrato de acero sumergido en el baño de la aleación binaria Al-Si, es preciso utilizar el diagrama ternario $\mathrm{Fe}-\mathrm{Al}-\mathrm{Si}$, contenido en la figura $3^{[16,17]}$. En la literatura ${ }^{[18,19]}$ se describe un método aproximado para predecir las diferentes capas que se pueden formar, basado en una variante de la regla de Rhines aplicada sobre una proyección de la superficie del líquidus en el diagrama ternario, en lugar del corte isotérmico que debería usarse pero que no se encuentra en la literatura. Se traza una línea recta, uniendo la composición del substrato (casi Fe puro) con la composición del baño líquido ( $\mathrm{Al}-25 \% \mathrm{Si}$ ) y las zonas de fase única atravesadas por esta línea indican qué fases pueden aparecer en la capa depositada sobre el substrato.

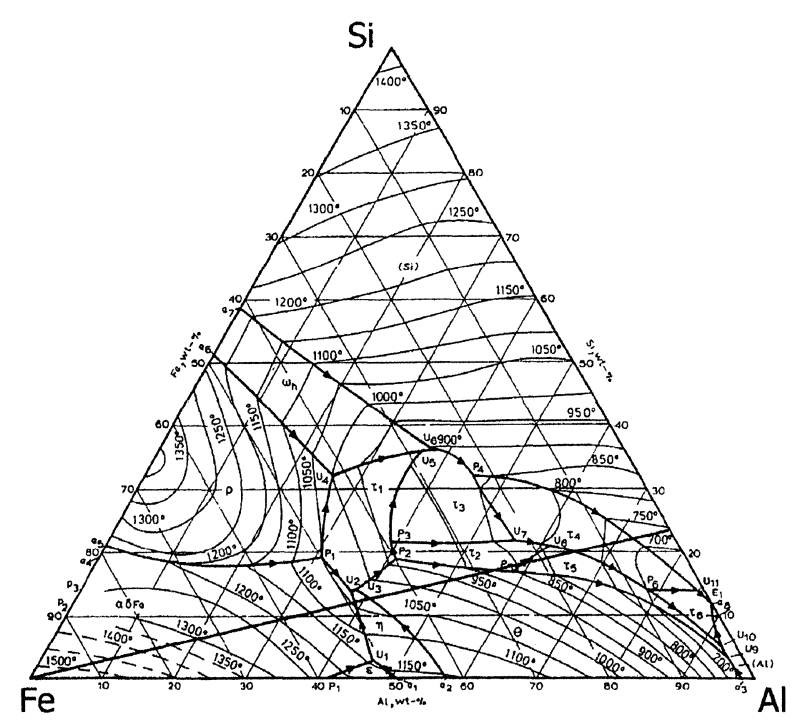

Figura 3. Aplicación de la regla de Rhines sobre un diagrama ternario $\mathrm{Fe}-\mathrm{Al}-\mathrm{Si}$.

Figure 3. Rhines rules in the ternary diagram Fe-Al-Si. Rev. Metal. Madrid 36 (2000) 
Evidentemente, la exactitud de la predicción depende de la exactitud del diagrama ternario usado $^{[18]}$.

De acuerdo a este procedimiento, las fases que pueden aparecer (a partir del substrato) son:

1. $\eta \quad \mathrm{Fe}_{2} \mathrm{Al}_{5}$

2. $\theta \quad \mathrm{FeAl}_{3}$

3. $\tau_{2} \quad \mathrm{Al}_{12} \mathrm{Fe}_{6} \mathrm{Si}_{5}$

4. $\tau_{5} \quad \mathrm{Fe}_{6} \mathrm{Al}_{15} \mathrm{Si}_{5}$

5. $\tau_{4} \quad \mathrm{Al}_{3} \mathrm{FeSi}_{2}$

6. Si primario y constituyente eutéctico ternario

Sin embargo, en las muestras analizadas, las fases $\theta\left(\mathrm{FeAl}_{3}\right)$ y $\tau_{5}\left(\mathrm{Fe}_{6} \mathrm{Al}_{15} \mathrm{Si}_{5}\right)$ no aparecen y el resto de las fases sólo aparece dependiendo del tiempo de inmersión y del recocido de difusión, de acuerdo a la aproximación de la regla de Rhines.

\subsection{Muestras con temperatura de precalenta- miento variable y sin recocido de difusión}

En esta serie de experimentos la única variable fue la temperatura de precalentamiento. Se precalentaron cinco muestras hasta temperaturas de 650, $700,750,800$ y $850^{\circ} \mathrm{C}$, respectivamente, para sumergirlas posteriormente en el baño líquido por 0,1 s y enfriarlas rápidamente con $\mathrm{N}_{2}$. No se aplicó recocido de difusión.

En todas las muestras analizadas sólo se observó la formación de tres capas, tal y como se aprecia en la figura 4. Dichas capas se han identificado como $\tau_{2}\left(\mathrm{Al}_{12} \mathrm{Fe}_{6} \mathrm{Si}_{5}\right)$, adyacente al substrato, $\tau_{4}\left(\mathrm{Al}_{3} \mathrm{FeSi}_{2}\right)$ y el eutéctico, donde a la vez aparecen zonas de silicio puro.

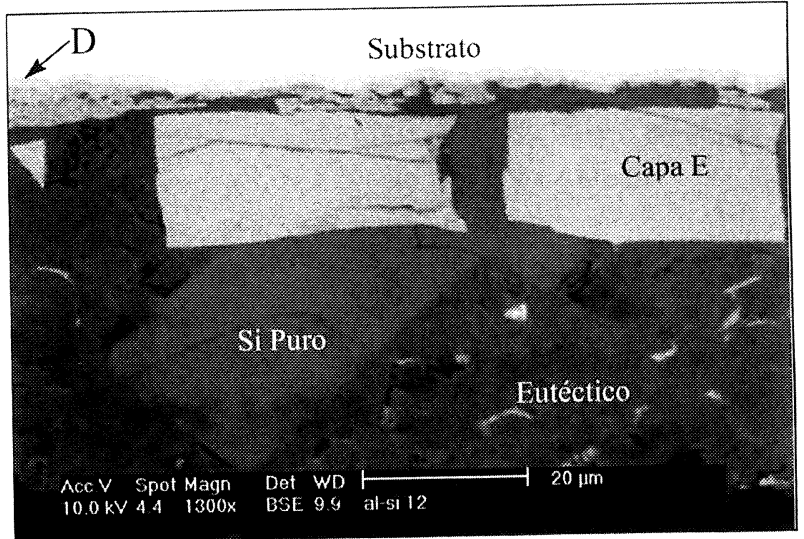

Figura 4. Capas formadas en la muestra precalentada a $800^{\circ} \mathrm{C}$. Capa A- $\tau_{2}$. Capa B- $\tau_{4}$.

Figure 4. Layers in the sample preheated at $800^{\circ} \mathrm{C}$. Layer $A-\tau_{2}$, layer $B-\tau_{4}$.
No se observó una clara influencia de la temperatura de precalentamiento sobre las capas formadas: solamente a temperaturas más altas, la capa adyacente al substrato $\tau_{2}$ deja de ser discontinua, convirtiéndose en una capa bien definida a partir de $800^{\circ} \mathrm{C}$. La cantidad de silicio depositado es similar en todas las muestras.

Si el tiempo de inmersión, en vez de $0,1 \mathrm{~s}$, se aumenta a $5 \mathrm{~s}$ en las muestras precalentadas a $850^{\circ} \mathrm{C}$, se observa la presencia de la fase ordenada $\mathrm{Fe}_{3} \mathrm{Si}\left(\mathrm{DO}_{3}\right)$ adyacente al substrato con un espesor aproximado de $1 \mu \mathrm{m}$, tal y como se aprecia en la figura 5 .

Al realizarse mediciones del espesor resultante del substrato y del espesor total de las muestras, incluyendo el recubrimiento y el substrato, (Fig. 6), se observó que la temperatura de precalentamiento no influye, prácticamente, en el espesor del substrato. Sin embargo, el espesor total

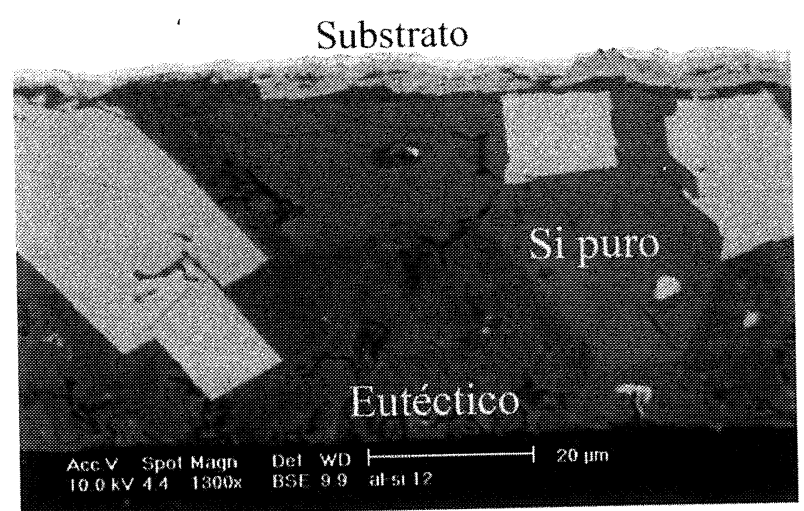

0.1 segundos

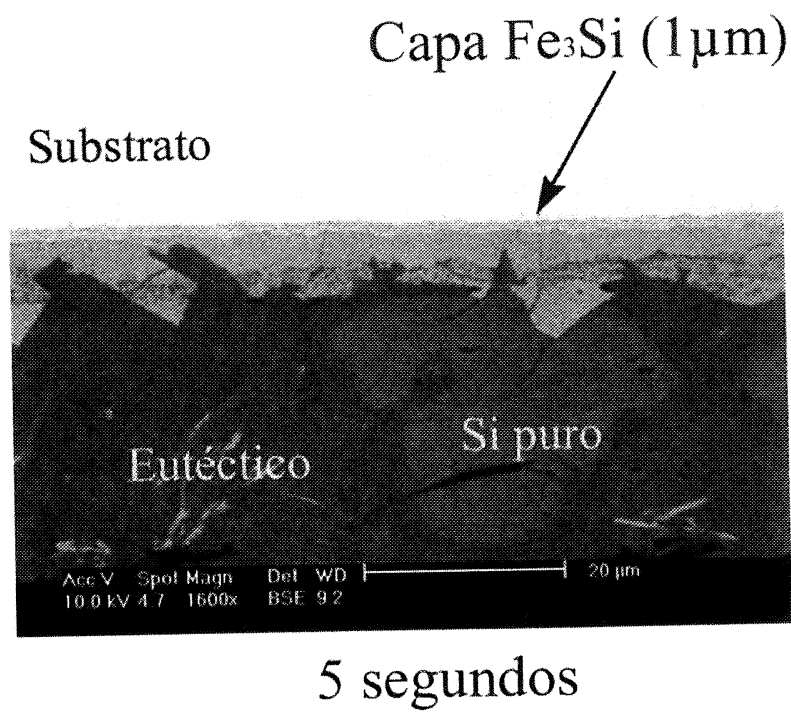

Figura 5. Capas depositadas con diferentes tiempos de inmersión en muestras sin recocido de difusión.

Figure 5. Deposited layers with different dipping times and without diffusion annealing. 


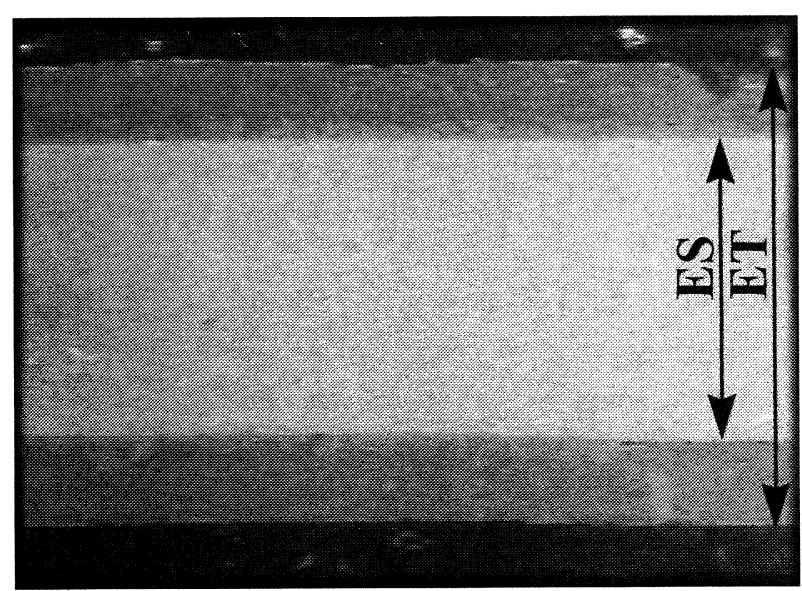

Figura 6. Espesor total (ET) y del substrato (ES) de una muestra.

Figure 6. Total thickness (ET) and substrate thickness (ES) in a sample.

aumenta, llegando a un máximo entre 700 y 750 ${ }^{\circ} \mathrm{C}$, disminuyendo posteriormente. En el gráfico de la figura 7 pueden apreciarse estas variaciones del espesor en función de la temperatura de precalentamiento.

\subsection{Muestras con tiempo de inmersión varia- ble y con recocido de difusión constante}

Las muestras se precalentaron a $850^{\circ} \mathrm{C}$ y se sumergieron en el baño durante diferentes tiempos: 2, 5 , 20, 100, 200 y 600 s. Posteriormente, se aplicó un recocido de difusión a $800^{\circ} \mathrm{C}$ durante 60 s, seguido de un enfriamiento a $20^{\circ} \mathrm{C} / \mathrm{min}$ hasta los 300 ${ }^{\circ} \mathrm{C}$ en el horno de infrarrojos, y otro final rápido en la cámara superior soplando $\mathrm{N}_{2}$.

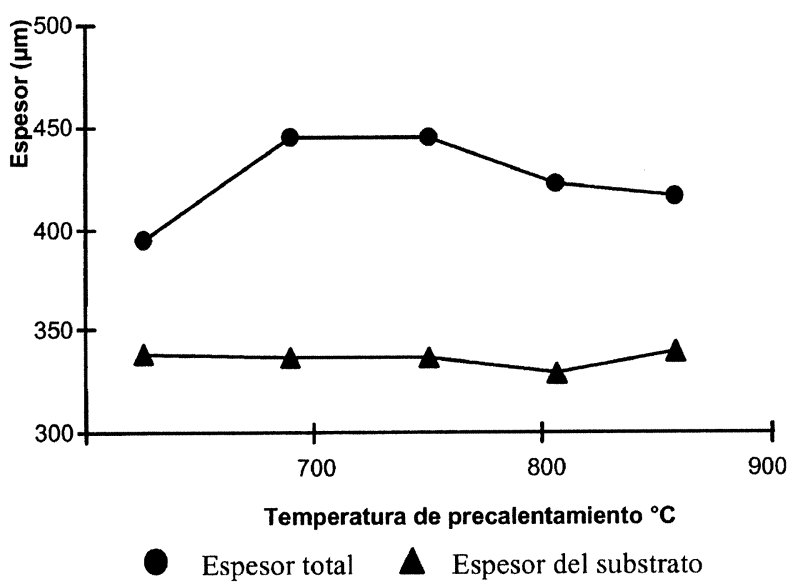

Figura 7. Comportamiento del espesor total y del substrato en función de la temperatura de precalentamiento (tiempo de inmersión $0,1 \mathrm{~s})$.

Figure 7. Total and substrate thickness vs. preheating temperature (dipping time $0.1 \mathrm{~s}$.)
La muestra sumergida durante $600 \mathrm{~s}$ en el baño se disolvió completamente. En el resto se formaron 6 capas, identificándose como:
A- Fase ordenada $\mathrm{DO}_{3}\left(\mathrm{Fe}_{3} \mathrm{Si}\right)$
B- $\zeta \mathrm{FeAl}_{2}$
C- $\tau_{1} \mathrm{Al}_{3} \mathrm{Fe}_{3} \mathrm{Si}_{2}$
D- $\tau_{2} \mathrm{Al}_{12} \mathrm{Fe}_{6} \mathrm{Si}_{5}$
E- $\tau_{4} \mathrm{Al}_{3} \mathrm{FeSi}_{2}$
F- Eutéctico

En la figura 8 se muestran las capas obtenidas después de las inmersiones durante 5,20 y $200 \mathrm{~s}$. Con el recocido de difusión aplicado, el silicio puro que se deposita después de la inmersión se difunde, formando en el substrato la fase ordenada $\mathrm{DO}_{3}$ (capa A). Esta capa uniforme aumenta de espesor con el tiempo de inmersión hasta los 100 s, manteniéndose luego aproximadamente constante con un espesor de $11 \mu \mathrm{m}$. El espesor promedio del constituyente eutéctico (capa F) disminuye con el aumento del tiempo de inmersión (de $30 \mu \mathrm{m}$ a $8 \mu \mathrm{m}$ ), manteniéndose constante a partir de los 20 s. Se observó también que $\tau_{4}$ (capa E) aumenta con el tiempo de inmersión y disminuye a partir de los $200 \mathrm{~s}$. En la figura 8 se observa una gráfica que describe los comportamientos de las capas mencionadas anteriormente. En esta serie de experimentos las zonas de silicio puro disminuyen considerablemente durante el recocido, debido a la difusión.

Las muestras con tiempo de inmersión de $5 \mathrm{~s}$ se compararon con muestras con igual tiempo de inmersión, pero sin recocido de difusión. Se pudo apreciar que la capa de la fase ordenada $\mathrm{Fe}_{3} \mathrm{Si}$ es muy pequeña cuando no se aplica recocido de difusión $(\sim 1 \mu \mathrm{m})$ y solo aparecen las capas $\tau_{2}, \tau_{4} \mathrm{y}$ el constituyente eutéctico junto con el silicio puro. Después del recocido de difusión el espesor de la capa de $\mathrm{Fe}_{3} \mathrm{Si}$ aumenta aproximadamente en 1,5 $\mu \mathrm{m}$ y se forman el resto de las capas.

El espesor original del substrato $(0,35 \mathrm{~mm})$ disminuye durante la inmersión porque parte del hierro se disuelve en el baño de Al-Si líquido. Al mismo tiempo, se forma una capa de productos ternarios Fe-Al-Si sobre el substrato. En la figura 9 se aprecia que la diferencia entre el espesor del substrato y el espesor total permanece constante: sólo el substrato se está disolviendo a través de la capa depositada (se disolvió completamente después de $600 \mathrm{~s}$ ). Un tiempo de inmersión superior a los $20 \mathrm{~s}$ no presenta ninguna ventaja en cuanto a espesor de capa depositada. 


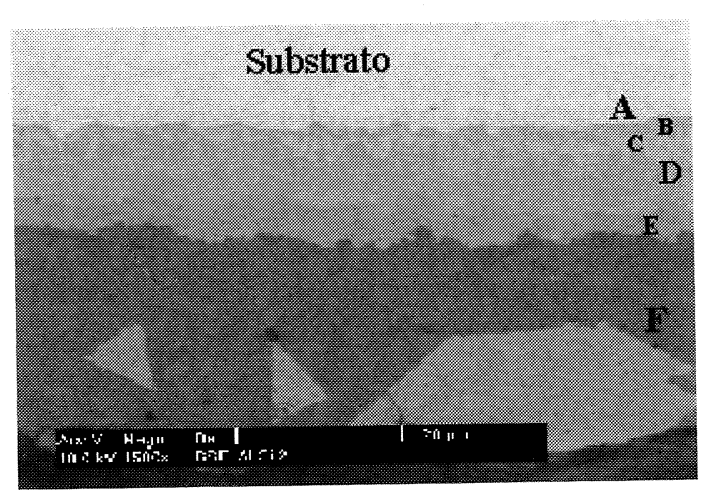

$5 \mathrm{sec}$

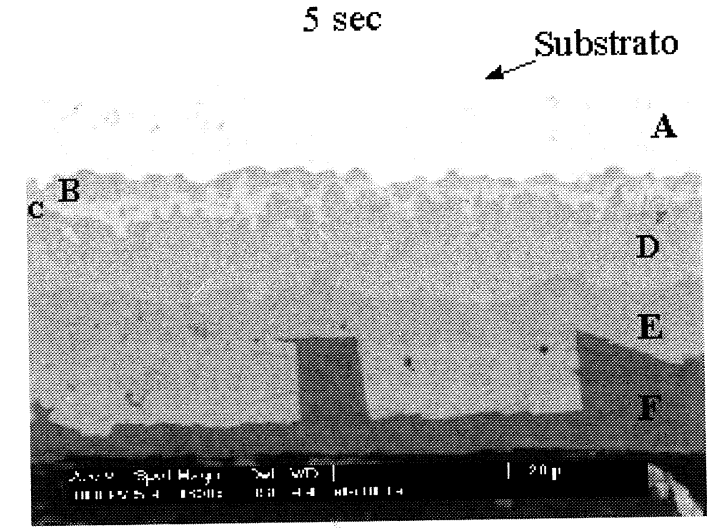

$200 \mathrm{sec}$

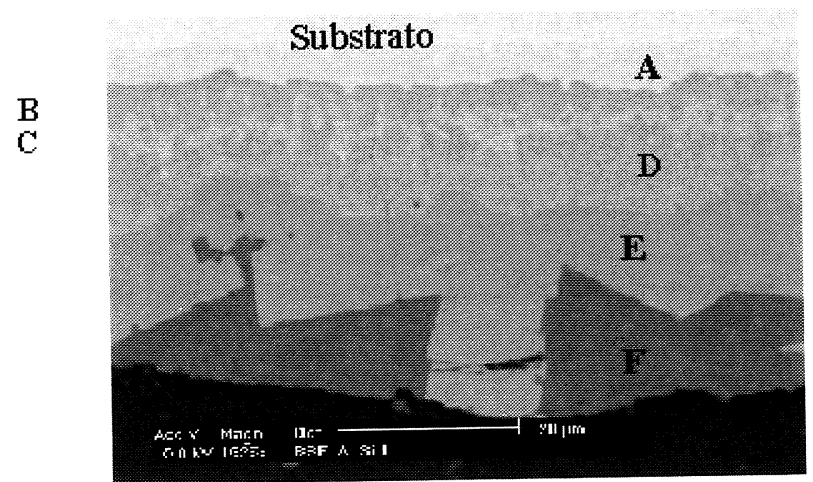

$20 \mathrm{sec}$

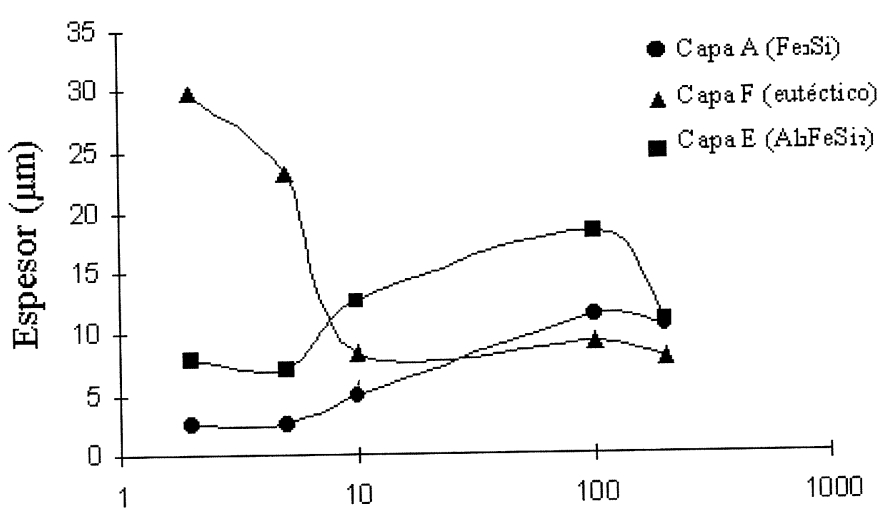

Tiempo de inmersión (s)

(escala $\log$.)

Figura 8. Aspecto de las capas depositadas después de diferentes tiempos de inmersión en el baño. En la gráfica se observa la variación del espesor de dos diferentes capas, en función del tiempo de inmersión.

Figure 8. Aspect of the coating affer different dipping times. The graph shows changes of thickness of the different phases vs. dipping times.

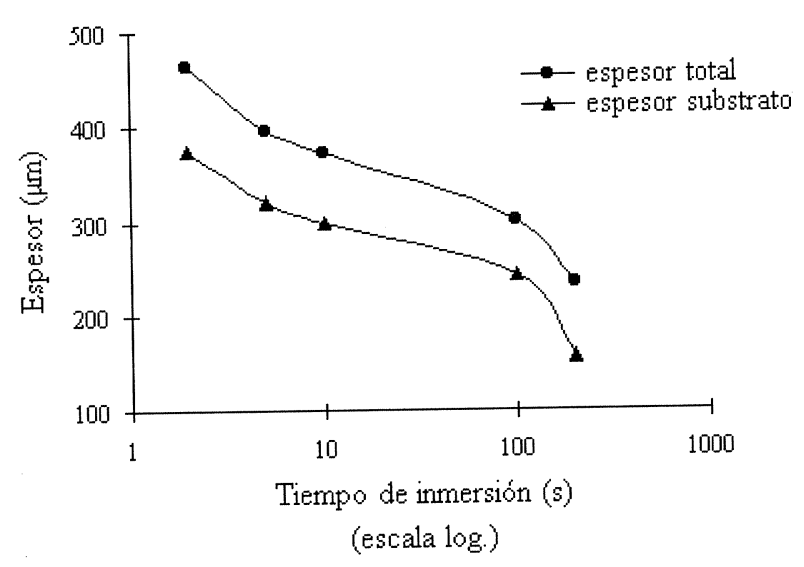

Figura 9. Variación del espesor total y del substrato en función del tiempo de inmersión de las muestras en el baño.

Figure 9. Changes in the substrate and total thickness vs. dipping time.

En cada muestra se hicieron perfiles de composición química para determinar la distribución del silicio, hierro y aluminio en las capas y en el substrato. La figura 10 muestra una gráfica donde se su- perponen 7 perfiles de silicio, de 40 puntos cada uno, en la muestra sumergida durante $20 \mathrm{~s}$. La imagen de la derecha muestra las zonas donde se midieron los perfiles. Es posible distinguir en el gráfico las diferentes capas según la concentración del silicio, correspondiéndose con las zonas identificadas en la fotografía.

\subsection{Muestras con temperaturas de recocido de difusión variable}

La figura 11 muestra la secuencia seguida en el experimento. Las muestras se precalentaron a $850^{\circ} \mathrm{C}$ y se sumergieron en el baño durante $5 \mathrm{~s}$; posteriormente se aplicó el recocido de difusión a 700 y 800 ${ }^{\circ} \mathrm{C}$, respectivamente, durante $60 \mathrm{~s}$, seguido de un enfriamiento controlado.

En la figura 12 se aprecian las diferencias entre las capas obtenidas. A temperaturas de $800^{\circ} \mathrm{C} \mathrm{el}$ espesor de la capa de $\mathrm{Fe}_{3} \mathrm{Si}$ es mayor, al igual que el espesor de la capa $\tau_{2}\left(\mathrm{Al}_{12} \mathrm{Fe}_{6} \mathrm{Si}_{5}\right)$, mientras que el espesor de la capa $\zeta\left(\mathrm{FeAl}_{2}\right)$ es ligeramente menor. 

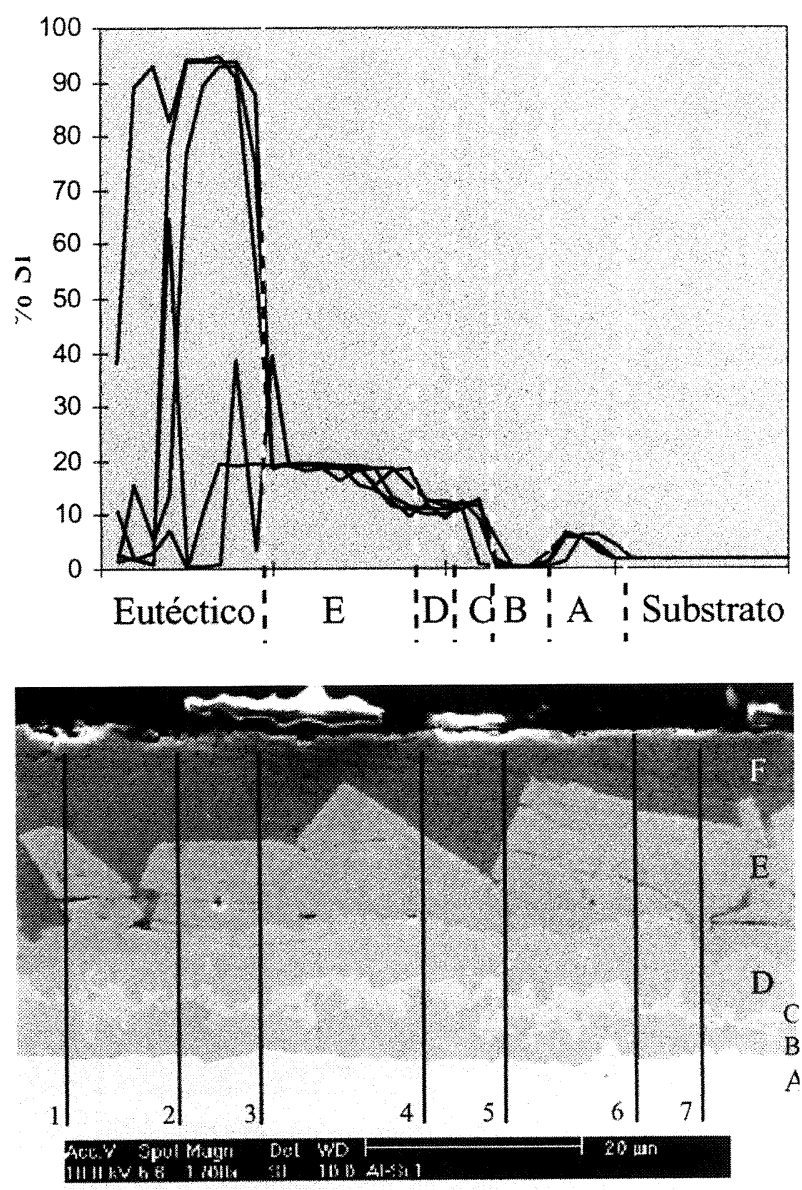

Figura 10. Perfiles de concentración de silicio en la muestra sumergida durante $20 \mathrm{~s}$.

Figure 10. Silicon concentration profiles in the sample dipped during $20 \mathrm{~s}$.

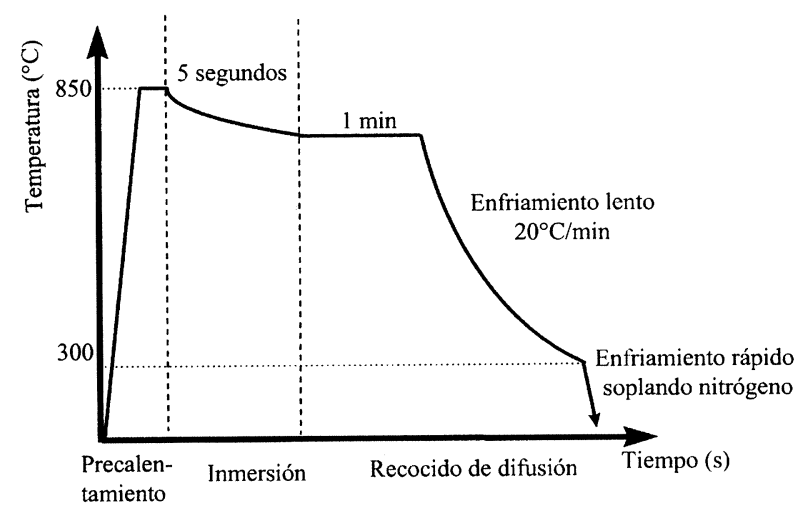

Figura 11. Tratamiento aplicado a las muestras con temperatura de recocido de 700 y $800{ }^{\circ} \mathrm{C}$.

Figure 11. Treatment applied to the samples annealed at 700 and $800^{\circ} \mathrm{C}$.

La tabla II muestra como varían los espesores promedio de cada capa, así como los espesores total y del substrato, que varían, en general, muy poco.
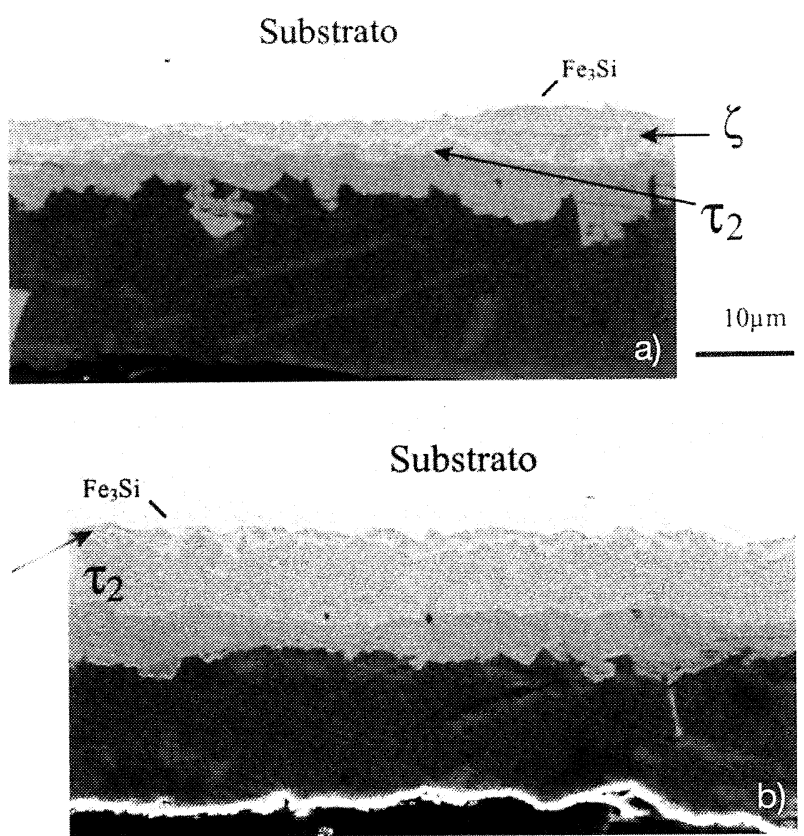

Figura 12. Aspecto de las capas obtenidas con recocido de difusión a $700^{\circ} \mathrm{C}$ (a) y a $800^{\circ} \mathrm{C}($ (b)

Figure 12. Aspect of the coating with diffusion annealing at $700{ }^{\circ} \mathrm{C}(\mathrm{a})$ and $800^{\circ} \mathrm{C}$ (b)

Tabla II. Espesores de las muestras recocidas a 700 y $800{ }^{\circ} \mathrm{C}$

Table II. Thickness of the samples annealed at 700 and $800^{\circ} \mathrm{C}$

\begin{tabular}{lccccc}
\hline Espesores $(\mu \mathrm{m})$ & total & substrato & $\mathrm{Fe}, \mathrm{Si}$ & $\zeta$ & $\tau_{2}$ \\
Recocido $700^{\circ} \mathrm{C}$ & 415 & 344 & 0.84 & 1.90 & 6.80 \\
Recocido $800^{\circ} \mathrm{C}$ & 395 & 321 & 2.60 & 1.15 & 7.60 \\
\hline
\end{tabular}

\section{Substrato}

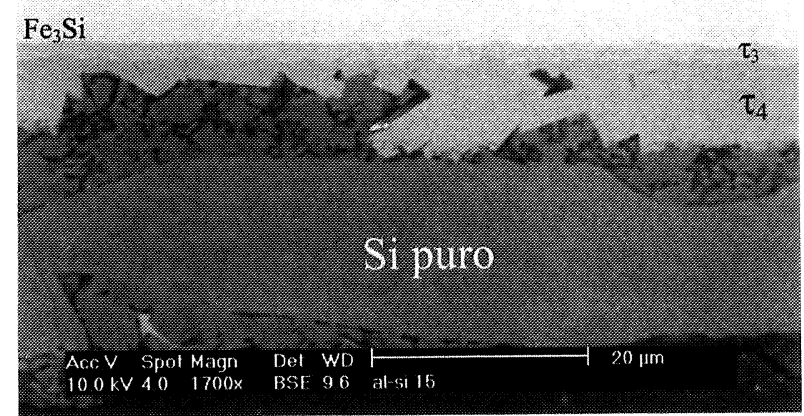

Figura 13. Aspecto de las capas obtenidas en las muestras con doble inmersión.

Figure 13. Aspect of the coating in the samples with double dipping.

Rev. Metal. Madrid 36 (2000) 


\subsection{Muestras con doble inmersión en el baño}

Se precalentaron una serie de muestras a $850^{\circ} \mathrm{C}$ y se sumergieron en el baño durante $20 \mathrm{~s}$, aplicándoseles posteriormente un recocido intermedio a temperaturas de 650,700 y $750^{\circ} \mathrm{C}$ durante $10 \mathrm{~s}$. A continuación se sumergieron nuevamente en el baño durante $0,1 \mathrm{~s}$, dejándolas enfriar lentamente durante $10 \mathrm{~s}$ en el horno de infrarrojos, proporcionándolas, a continuación, un enfriamiento rápido con nitrógeno en la cámara superior.

Se observaron cuatro capas identificadas como: fase ordenada $\mathrm{Fe}_{3} \mathrm{Si}, \tau_{3}, \tau_{4}$ y constituyente eutéctico, donde aparecen grandes zonas de silicio puro, tal y como se aprecia en la figura 13. Las muestras con recocido intermedio de 700 y $750^{\circ} \mathrm{C}$ no mostraron diferencias en cuanto al espesor de las capas, el espesor total de las muestras y la cantidad de silicio puro. Sin embargo, la muestra recocida a $650^{\circ} \mathrm{C}$ presenta una capa de eutéctica mucho mayor: $47 \mu \mathrm{m}$ de espesor en comparación con $27 \mu \mathrm{m}$ de espesor en las anteriores. La cantidad de silicio puro también es superior.

En comparación con las muestras posteriormente sumergidas solo una vez en el baño y a las que, posteriormente, se aplicó un recocido de difusión, en estas muestras doblemente sumergidas, se observa, claramente, una gran cantidad de silicio puro aún sin difundir y a la vez una capa de la fase $\mathrm{Fe}_{3} \mathrm{Si}$.

\section{CONCLUSIONES}

En todas las muestras estudiadas se observan fases intermetálicas de acuerdo al diagrama ternario Al$\mathrm{Fe}-\mathrm{Si}$, las cuales aparecen dependiendo del tiempo de inmersión en el baño y del recocido de difusión posterior. En las muestras sin recocido solo aparecen tres capas, así como silicio puro en la capa eutéctica. Al aumentar el tiempo de inmersión se forma una capa de la fase ordenada $\mathrm{Fe}_{3} \mathrm{Si}$ debido a la difusión del silicio. Por su parte, la temperatura de precalentamiento de la muestras no evidencia tener influencia sobre las capas formadas, apreciándose, únicamente, que a la temperatura de 800 ${ }^{\circ} \mathrm{C}$ la capa $\tau_{2}$ deja de ser discontinua.

Con el recocido de difusión aumenta el número de capas, debido a la difusión del silicio puro depositado después de la inmersión en el baño, y aparece la capa de la fase ordenada $\mathrm{Fe}_{3} \mathrm{Si}$. Esta capa aumenta de espesor con el tiempo de inmersión, llegando a ser constante a partir de los $100 \mathrm{~s}$. La cantidad de silicio puro en la capa eutéctica, después del recocido, es mínima.

Por doble inmersión se obtienen grandes áreas de silicio puro en la capa depositada que, con un recocido posterior, podrían difundirse en el substrato.

Se logró un cierto grado de difusión de silicio en el substrato, formándose una capa (A) de $\mathrm{Fe}_{3} \mathrm{Si}$, con concentración fija de $25 \%$ atómico de silicio. El espesor de esta capa aumenta con el tiempo de inmersión y se lograron espesores hasta de $11 \mu \mathrm{m}$. Ensayos posteriores de recocido prolongado demostrarán en qué medida es posible aumentar este espesor. De todos modos, cálculos teóricos demuestran que, aún cuando no se logre una concentración homogénea en todo el espesor, los gradientes en silicio también mejoran, considerablemente ${ }^{[16]}$, las propiedades magnéticas.

\section{REFERENCIAS}

[1] A.J. Moses, IEE Proceedings, 137-A 5 (1990) 233-245.

[2] M.F. LiTTMAnN, IEEE Trans. Magn. MAG-7 (1971) 48-60.

[3] Y. TaKada, J. Appl. Phys. 64 (1988) 53-67.

[4] M.F. LitTMann, J. Magn. Magn. Mater. 26 (1982) 1-10.

[5] Program Brite-EuRam III, Warm working for high silicon steel, BRPR - CT98 - 0727, 1998.

[6] T. Ros, Y. Houbaert, O. Fischer y J. Schneider, Conf. Abstract Proc. EMMA 2000, Kiev, Ukrania, 2000, p.168.

[7] A.J. Moses y G. Thursby, J. Mater. Sci.18 (1983 16571665.

[8] A.J. Moses y G. Thursby, J. Mater. Sci.18 (1983 16501656.

[9] H. Haijl, K. Okada, T. Hiratami y M. Ninomiya, J. Magn. Magn. Mater. 160, (1996) 109-114.

[10] M. IsHIKAWA, NKK Technical Review 72 (1995).

[11] K. OKadA, T. YAMAJI y K. KASAI, ISIJ Int. 36 (1996) 706 713.

[12] E. Smet, G. Krielaart y Y. Houbaert, Conf. Abstract Proc. EMMA, Zaragoza, España 1998, p 245.

[13] T. Ros, M. Prado, H. Vandriessche y Y. Houbaert, Conf. Abstract Proc. EMMA 2000, Kiev, Ukrania, 2000, p. 167.

[14] Y. Houbaert, T. Ros, H. Vandriessche y B.C. DE Cooman, Conf. Abstract Proc. EMMA 2000, Kiev, Ukrania, 2000, p. 167.

[15] T. Ros, Y. Houbaert y M. Prado, Proc. TRatermat 2000, Barcelona, España, 2000, A. Herrero y F. Vivas (Eds.), 2000, pp. 389-396.

[16] T. KozAKaI y T. MiYAZAKI, ISIJ Int. 34 (1994) 373-383.

[17] L. Dupré, S. Jacobs y J. Melkebeek aceptado para publicación en IEEE Trans. Magn., Septiembre 2000. 